\title{
Modern Diagnostic Methods in Power Transformers, Based on Dielectric Polarization
}

\author{
Wilder Herrera Portilla ${ }^{1}$, Guillermo Aponte Mayor ${ }^{2}$, Juan Carlos Burgos ${ }^{3}$, Belén Garcia ${ }^{4}$ \\ ${ }^{1,2}$ Grupo de Investigación en Alta Tensión - GRALTA. Universidad del Valle, Cali - Colombia. \\ ${ }^{3,4}$ Grupo Diamat Universidad Carlos III - Madrid - España.
}

Email: wilderh6@yahoo.com,gponte@univalle.edu.co,jcburgos@ing.uc3m.es, bgarciad@ing.uc3m.es

Received: November $10^{\text {th }}, 2017$.

Accepted: November $21^{\text {th }}, 2017$.

Published:.December 13 $3^{\text {th }}, 2017$.

Copyright $\odot 2016$ by authors and Institute of Technology Galileo of Amazon (ITEGAM)

This work is licensed under the Creative

Commons Attribution International

License (CC BY 4.0).

http://creativecommons.org/licenses/by/4.0/

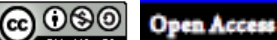

\begin{abstract}
In this article some modern diagnostic methods are presented, which based on the Dielectric Response of the insulation, help to estimate the condition of the transformer. These methods are comparative tools, which allow to estimate the moisture content of the insulation, as well as to perceive trends in its degradation.
\end{abstract}

Keywords: Power Transformers, Dielectric Polarization, Modern diagnostic.

\section{Métodos Modernos de Diagnostico en Transformadores de Potencia, Basados en la Polarización Dieléctrica}

\section{RESUMEN}

En este articulo se presentan algunos métodos modernos de diagnóstico, que basados en la Respuesta Dieléctrica del aislamiento, ayudan a estimar la condición del transformador. Estos métodos son herramientas comparativas, que permiten estimar el contenido en humedad del aislamiento, así como percibir tendencias en su degradación.

Palabras Claves: Transformadores de Potencia, Polarización Dieléctrica, Modernos de diagnóstico.

\section{INTRODUCCIÓN}

El transformador de potencia es uno de los elementos más costosos del sistema de potencia, siendo generalmente muy confiable, con una vida útil de 20 a 35 años, que puede extenderse hasta unos 60 años con un mantenimiento apropiado [1].

Actualmente un alto porcentaje de los transformadores instalados en los sistemas eléctricos, se encuentra en una etapa de envejecimiento avanzada, por lo que se hace necesario conocer su condición a través de pruebas de diagnostico y programas de mantenimiento, para detectar situaciones de riesgo o de limitación de uso, que podrían llevar a tomar acciones para prevenir la ocurrencia de fallas graves, con las consiguientes interrupciones del servicio, o programar acciones que permitan prolongar la vida del transformador (tales como secados).

Uno de los elementos que determinan la vida útil remanente del transformador es su aislamiento, el cual se divide en aislamiento líquido (generalmente aceite) y aislamiento solido (papel).

Durante su funcionamiento, los transformadores están sometidos a condiciones que deterioran su aislamiento, las cuales se originan generalmente por alta temperatura y presencia humedad, que combinadas con esfuerzos eléctricos y mecánicos aceleran el proceso de degradación.

La degradación del aislamiento es por lo tanto una preocupación muy importante para las empresas que operan los transformadores. En años recientes han aparecido nuevos métodos de diagnostico, que basados en los cambios de la respuesta dieléctrica del aislamiento permiten estimar de manera indirecta su contenido de humedad y brindan información de la degradación de sus componentes. Los métodos de diagnostico que se presentan, basados en la respuesta dieléctrica, son de dos tipos:

En el dominio del tempo:

- Medición de la Tensión de Recuperación (RVM).

- Medición de Corriente de Polarización y Depolarización (PDC).

En el dominio de la frecuencia:

- Espectroscopia en el Dominio de la Frecuencia (FDS). 


\section{RESPUESTA DIELÉCTRICA EN EL DOMINIO DEL TIEMPO}

Cuando un material dieléctrico es sometido a un campo eléctrico estático, se desarrollan tres procesos fundamentales: el de carga, el de conducción (o de fuga) y el de polarización (Figura 1).

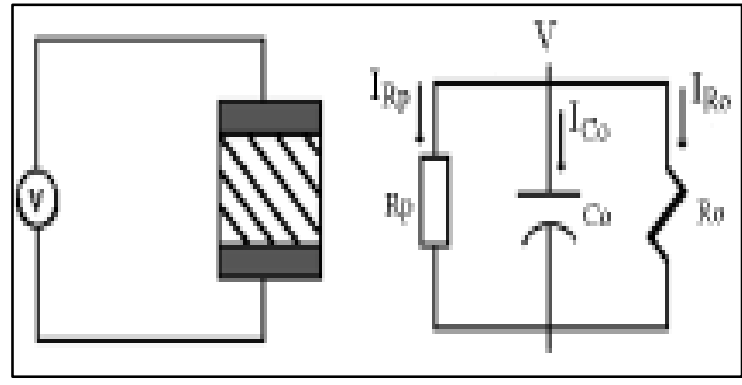

Figura 1: Modelo del aislamiento en DC.

Fuente: Autores, (2017).

ICo es la corriente que aparece como consecuencia de la carga de la capacitancia geométrica del aislamiento que separa las partes energizadas. Es una corriente de muy corta duración, que llega a cero cuando se carga completamente la capacitancia.

IRo es la corriente de conducción que fluye a través del volumen del material, es estable en el tiempo y proporcional a V/Ro.

IRp es la corriente que aparece como consecuencia del proceso de polarización del material aislante. La corriente debida a este fenómeno disminuye en el tiempo, pudiendo en los transformadores durar muchos minutos e incluso horas. La corriente de conducción y la de polarización, pueden ser caracterizadas por una sola cantidad, la conductividad específica.

La corriente de polarización, se genera debido a una densidad volumétrica de carga producida en los dipolos de los materiales dieléctricos. Cuando no existe un campo eléctrico externo, los dipolos individuales del material dieléctrico, están orientados de manera aleatoria. Un campo eléctrico aplicado, ejerce un par de torsión sobre los dipolos, que tiende alinearlos con el campo, en un mayor o menor grado. Como resultado de esta polarización, el dieléctrico está sometido a una tensión, almacenando energía, la cual quedará disponible al retirar el campo eléctrico.

Teniendo en cuenta los tres fenómenos anteriores, el comportamiento de un condensador con un dieléctrico polar puede modelarse como se muestra en la Figura 2, donde Co es la capacitancia geométrica, Ro es la resistencia de aislamiento y las redes Rpi-Cpi representan los distintos procesos de polarización que aparecen en el material con constantes de tiempo $\mathrm{Ti}=\mathrm{RiCi}$ [2].

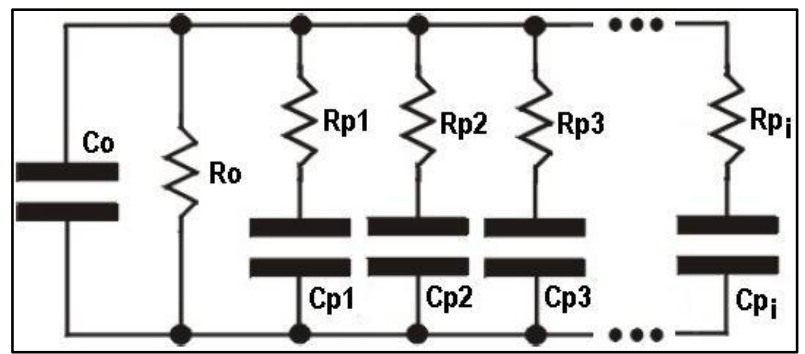

Figura 2: Modelo con los dipolos del aislamiento. Fuente: Autores, (2017).

\section{II.1 MEDICIÓN DE LA TENSIÓN DE RECUPERACIÓN RVM}

El método de la medición de la tensión de recuperación RVM (Recovery voltage measurements) consiste en la ejecución de varios ciclos de carga y descarga del dieléctrico, con cuyo resultado se construye un espectro de puntos llamado espectro de polarización. El espectro de polarización contiene tanto información de la humedad en el transformador como de la degradación de sus componentes aislantes.

Considerando el circuito de la Figura 3, inicialmente se le aplica al dieléctrico (el cual se encuentra descargado) un voltaje continuo Uc, durante un periodo de tiempo tc, mediante el cierre del interruptor S1. Durante este periodo la capacitancia Co se carga y se producen los procesos elementales de polarización con constantes de tiempo menores a tc.

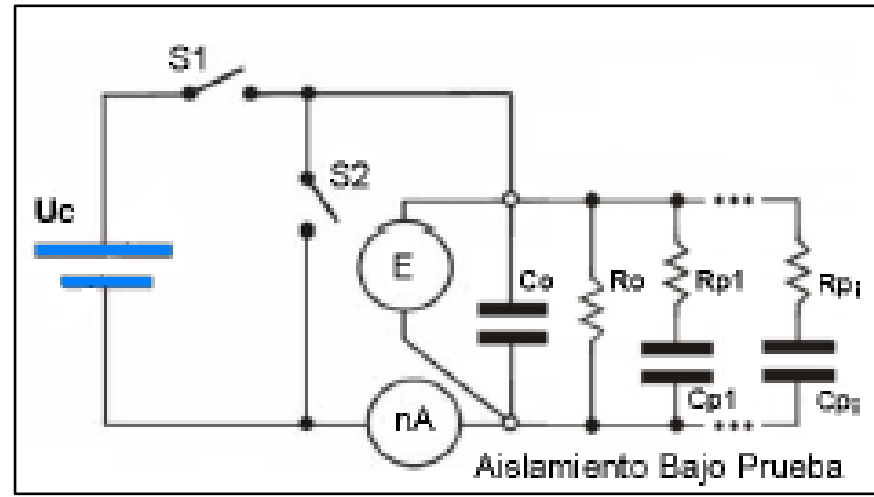

Figura 3: Circuito para la medición RVM.

Fuente: Autores, (2017).

Luego el aislamiento es separado de la fuente de voltaje por la apertura del interruptor S1 y es enseguida cortocircuitado por el cierre del interruptor S2, por un periodo de tiempo td. En esta etapa la capacitancia Co se descarga, y los procesos de polarización con constantes de tiempo más cortas (menores a td) se relajan.

Finalmente se abre el interruptor S2, en este instante quedan aun activados los procesos de polarización con constantes de tiempo tpi comprendidos entre tc y td $(\mathbf{t d}<\mathbf{t p i}<\mathbf{t c})$.

Como resultado del procedimiento anterior se obtiene la respuesta correspondiente a la descarga de los procesos elementales de polarización activados, lo que provoca la aparición de un voltaje entre los electrodos del dieléctrico, llamado voltaje de recuperación, mostrado en la Figura 4.

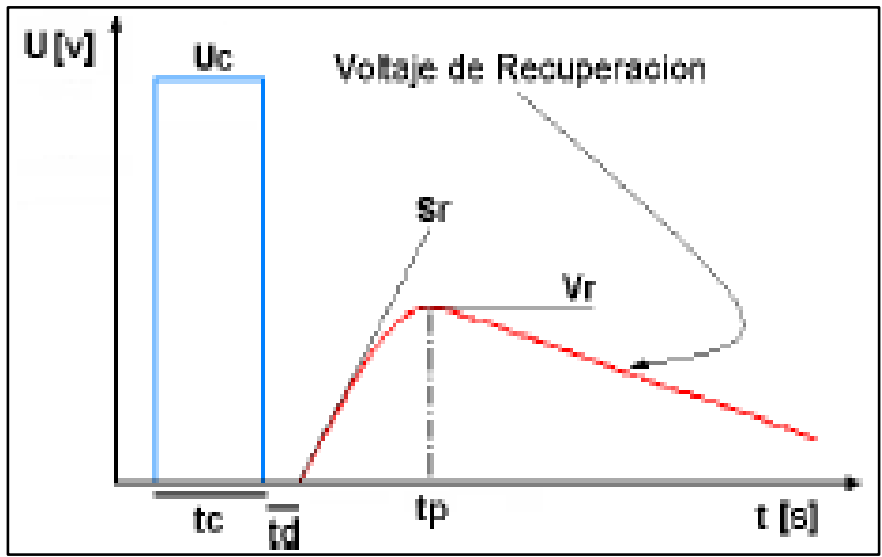

Figura 4: Voltaje de recuperación.

Fuente: Autores, (2017). 
El procedimiento se repite para completar una serie de ciclos de medida con diferentes tiempos de carga en un rango de $10 \mathrm{~ms}$ a $10.000 \mathrm{~s}$, manteniendo la relación $\mathrm{tc} / \mathrm{td}=2$.

De cada ciclo de carga y descarga se obtienen tres valores: el valor máximo del voltaje de recuperación $\mathrm{Vr}$, la pendiente inicial $\mathrm{Sr}$ y el tiempo al cual ocurre el pico máximo tp. Cada punto del espectro de polarización se obtiene ubicando el pico de la tensión de recuperación frente al tiempo de carga tc.

El tiempo de carga al cual ocurre el máximo pico del espectro de polarización se denomina la constante de tiempo central dominante Dominante $\mathrm{tc}_{\text {Dominante }}$.

\section{II.1.1 INFLUENCIA DE LA HUMEDAD Y EL ENVEJECIMIENTOSOBRE EL ESPECTRO DE POLARIZACIÓN}

En la Figura 5, se muestran varios espectros RVM correspondientes a diferentes contenidos de humedad, donde puede observarse que los máximos picos se desplazan a tempo más cortos, con el incremento de humedad [3].

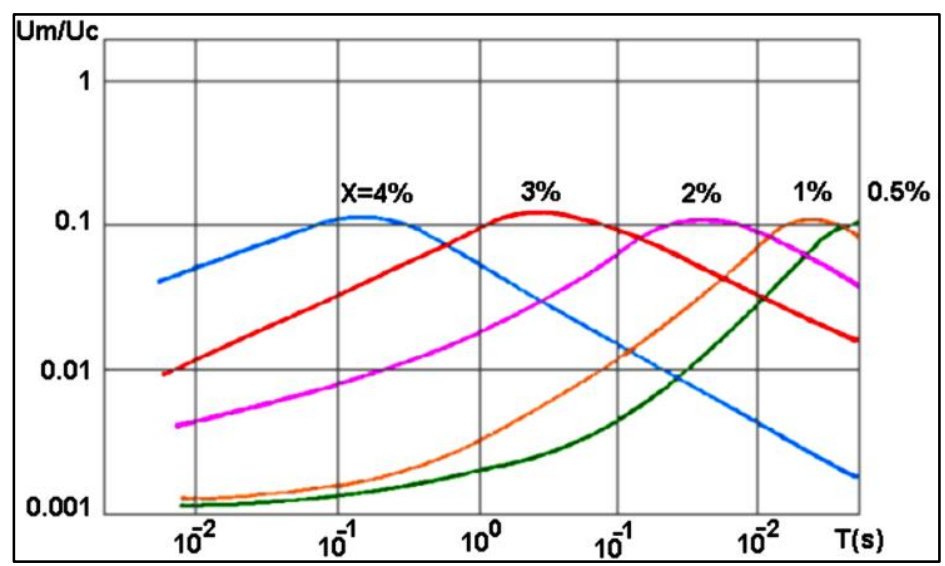

Figura 5: Dependencia del espectro de polarización con el grado de Humedad.

Fuente: Autores, (2017).

En referencia [3] se presenta la relación entre la constante de tiempo central dominante y la humedad del papel, lo cual es mostrado en la Figura 6, para diferentes valores de temperatura. Mediante esta relación puede estimarse el contenido de humedad del papel.

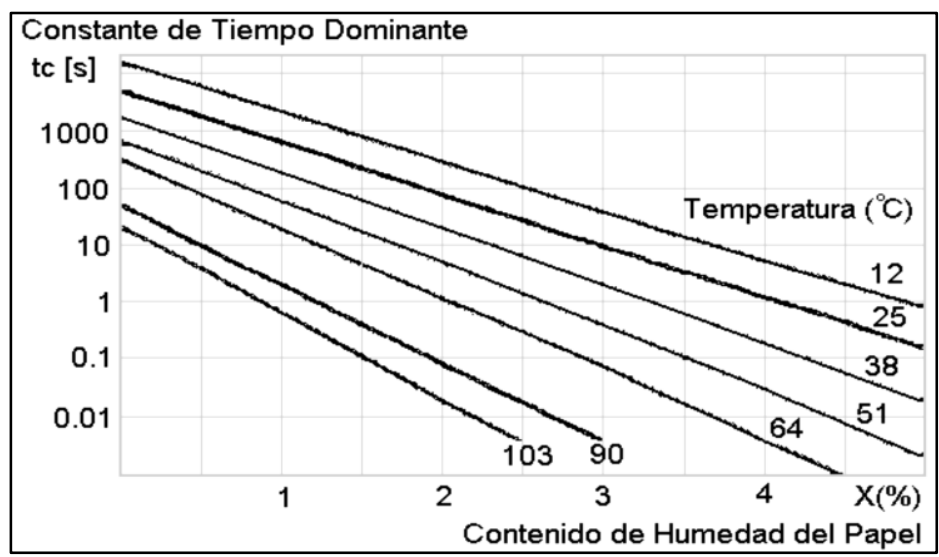

Figura 6: Estimación del contenido de humedad.

Fuente: Autores, (2017).
La constante de tiempo central dominante, decrece con el grado de envejecimiento del transformador, sin embargo, los procesos de envejecimiento generan humedad, por lo que el envejecimiento y la humedad están estrechamente ligados. La relación entre el envejecimiento y la humedad hace difícil separar la influencia de ambos factores, y por lo tanto obtener una interpretación precisa del grado de envejecimiento a partir de las medidas de respuesta dieléctrica.

En la Figura 7 se muestran las diferencias en los espectros de polarización de transformadores con distintas edades [4] Como se puede observar el aumento de la humedad y el envejecimiento del papel provocan un desplazamiento de la curva en el mismo sentido.

La temperatura del transformador durante la medición del voltaje de recuperación es un parámetro muy importante, ya que puede ocasionar desplazamientos significativos en los picos del espectro RVM. A temperaturas altas, el pico del espectro RVM se desplaza a tiempos más cortos.

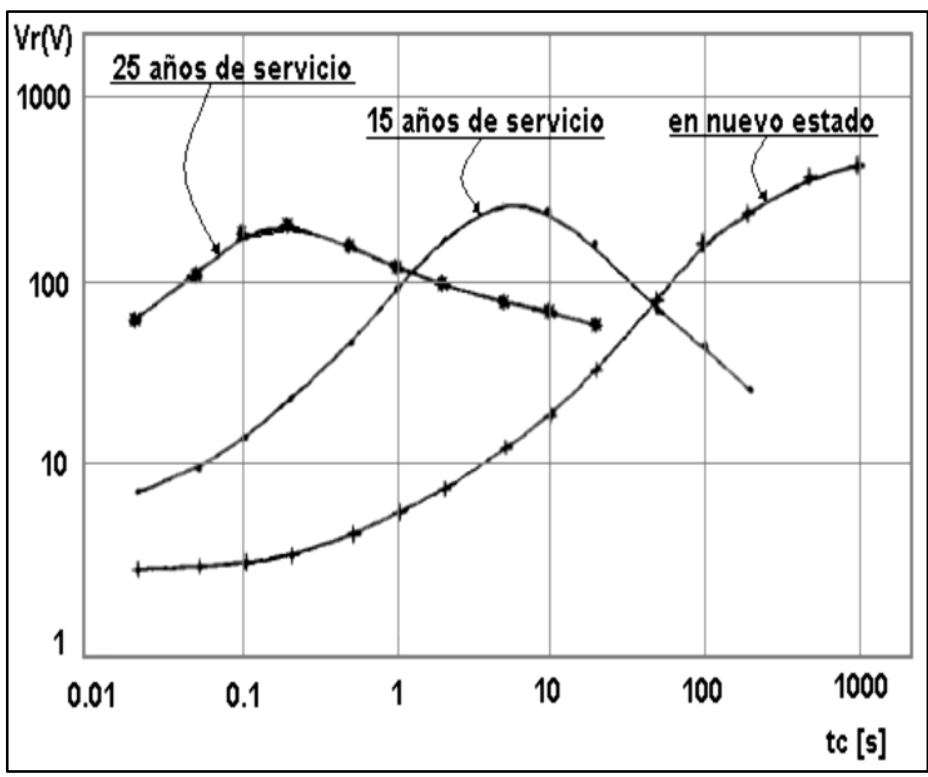

Figura 7: Curvas RVM de transformadores de edades diferentes.

Fuente: Autores, (2017).

\section{II.1.2 ANÁLISIS DE RESULTADOS DE RVM}

El diagnostico RVM, se puede realizar a través del análisis del espectro RVM, el cual provee un valor numérico para el contenido de humedad del papel.

Además resulta útil comparar los espectros RVM obtenidos en diferentes tiempos de vida del transformador, en este caso el análisis permite percibir tendencias en la degradación del aislamiento.

El grupo de trabajo de CIGRÉ TF15.01.09. en [13] alerta sobre las interpretaciones erróneas a las que puede dar lugar el análisis del espectro de polarización. En su opinión, esta interpretación es muy simplista y no considera parámetros importantes como la conductividad del aceite. En particular alertan sobre la obtención de contenidos de humedad excesivamente altos al interpretar las medidas RVM tomadas sobre transformadores nuevos, en los que el papel y el aceite no hayan alcanzado aún el equilibrio. 


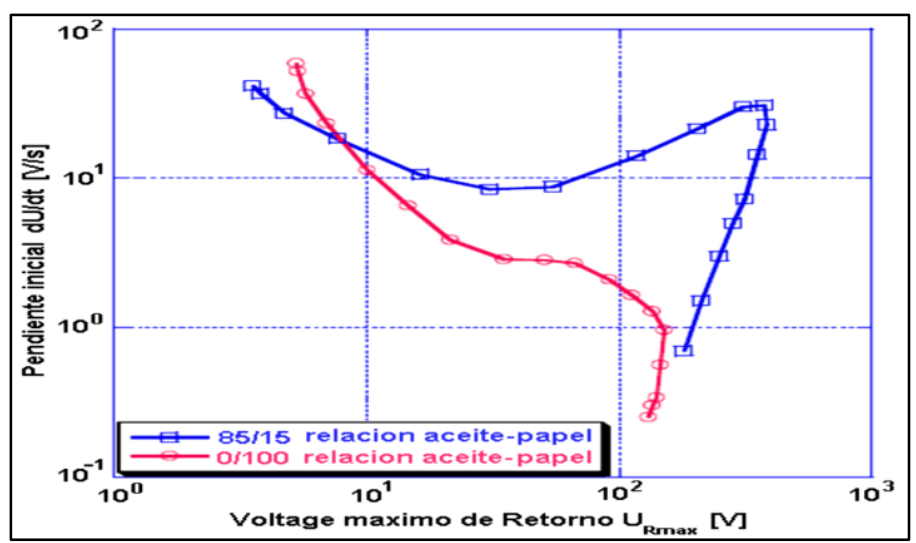

Figura 8: Representación de Guuinic.

Fuente: Tomado de [13].

A la vista de lo anterior, el grupo de trabajo de Cigré propuso una interpretación alternativa llamada representación de Guuinic que consiste en representar la pendiente inicial del espectro de polarización frente al valor pico del espectro de polarización, como se muestra en la figura 8 [13] (FIG 6BIS). Para obtener una estimación del contenido en humedad se propone comparar la forma de este gráfico con unos patrones y completar el estudio con el análisis del estado del aceite.

\subsection{MEDICIÓN DE LA CORRIENTE DE POLARIZACIÓN Y DEPOLARIZACIÓN PDC}

En la medición de la corriente de polarización y despolarización PDC (polarization and depolarization currents), se aplica un voltaje DC al aislamiento bajo prueba por un largo periodo tc (típicamente 104s), durante el cual fluye una corriente debido a los procesos de polarización. Luego el voltaje es retirado y el dieléctrico es cortocircuitado. La relajación de los procesos de polarización ya activados, produce una corriente en dirección opuesta, llamada la corriente de depolarización cuando los dipolos retornan a su posición de equilibrio.

En la Figura 9 se muestra el diagrama para la medición PDC, junto con las corrientes de polarización y despolarización, producidas por un escalón de voltaje de magnitud Uo. Estas corrientes están influenciadas por las propiedades de los materiales aislantes dentro del transformador y por la estrutura geométrica del sistema de aislamiento [5].
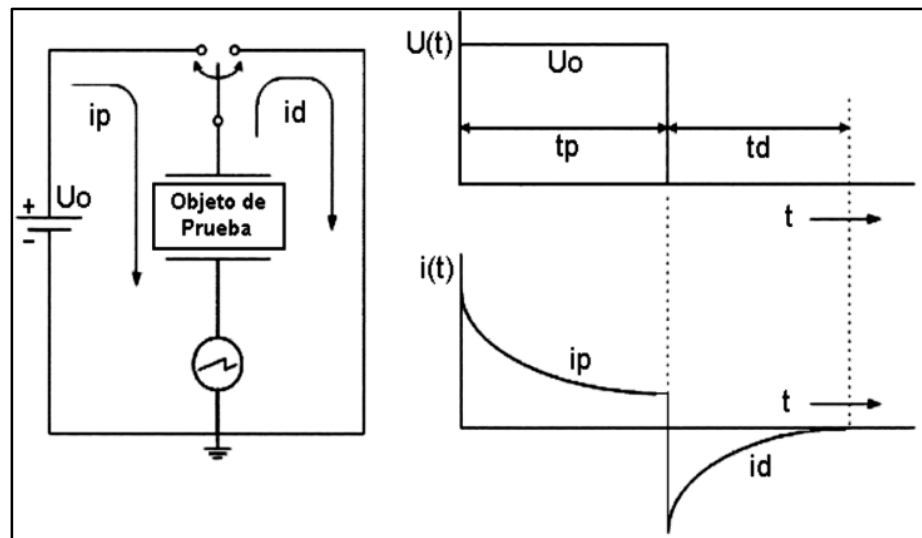

Figura 9: Corrientes de polarización y depolarización.

Fuente: Autores, (2017).

\section{II.2.1 INFLUENCIA DE LA CONDUCTIVIDAD DEL ACEITE Y EL PAPEL SOBRE LAS MEDICIONES PDC}

La naturaleza de las corrientes de polarización y despolarización dependen de varios factores incluyendo, la temperatura, la geometría, el contenido de humedad, la conductividad y condiciones de envejecimiento del aislamiento compuesto aceite/papel. Los cambios en la conductividad del papel aislante, afectan el final de la curva de la corriente de polarización. En la Figura 10 se muestra la variación del final de las curvas, para diferentes conductividades del papel [6].

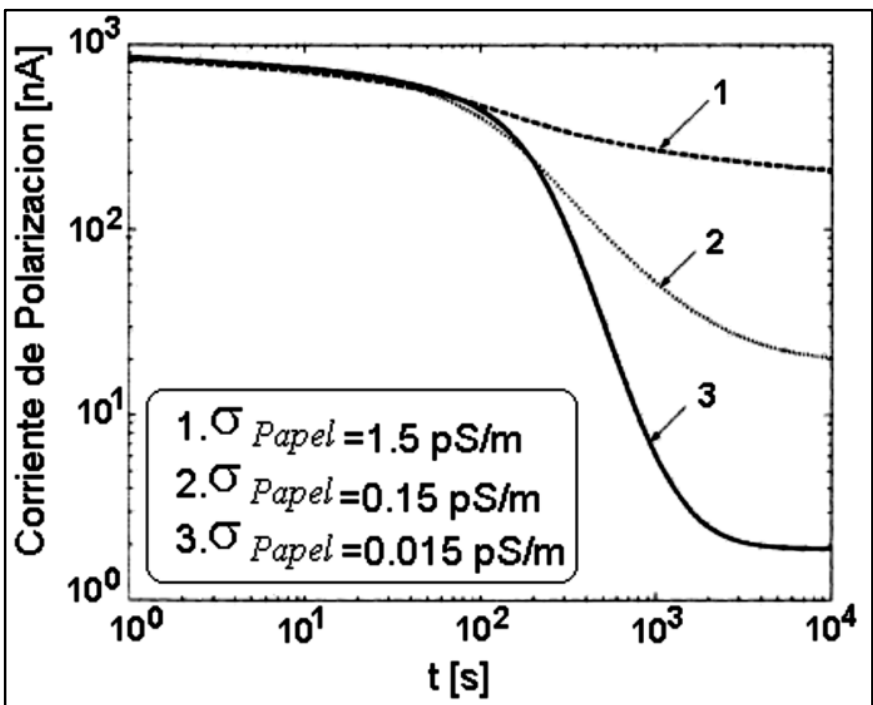

Figura 10: Variaciones de la corriente de polarización para varias conductividades del papel aislante.

Fuente: Autores, (2017).

Por otro lado la parte inicial de las corrientes de polarización y despolarización, están afectadas por las propiedades del aceite. En la Figura 11 se muestra la variación de la parte inicial de estas corrientes, para diferentes conductividades del aceite [6].

La polarización se comporta igual ante variaciones de la conductividad y ante variaciones de la humedad, cuanto mayor es la humedad mas altas son las corrientes de polarización y despolarización y aumenta la diferencia entre estas corrientes.

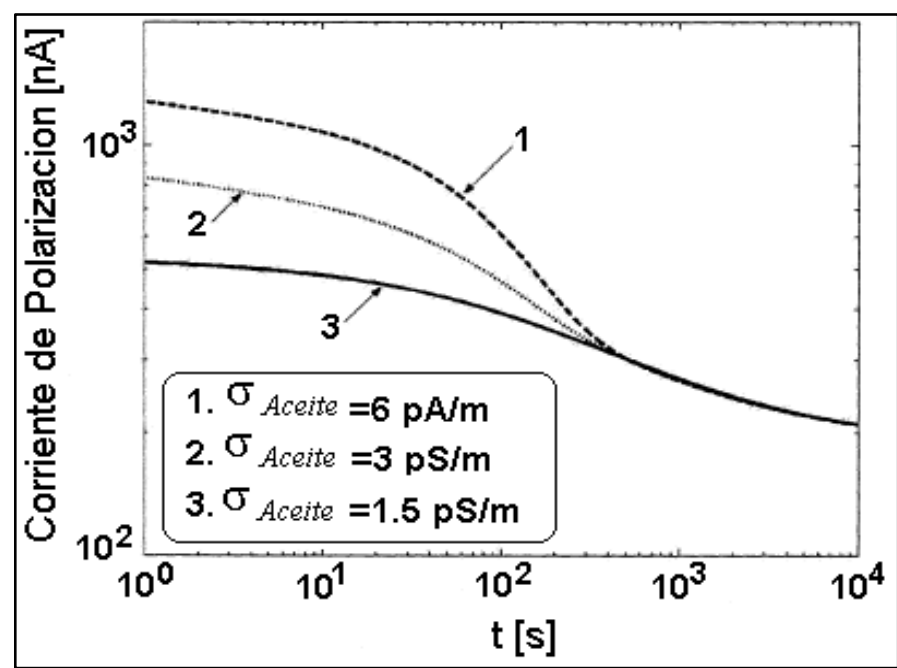

Figura 11: Variaciones de la corriente de polarización para varias conductividades del aceite.

Fuente: Autores, (2017). 
El envejecimiento también afecta la respuesta PDC, en parte debido a que se aumenta la humedad del papel. En la Figura 12 se muestran diferencias de la corriente de polarización, para varios contenidos de humedad del papel.

Como en los otros los métodos dieléctricos, la temperatura del transformador durante la medición PDC es un parámetro muy importante, ya que causa un desplazamiento de las curvas de las corrientes, aumentando su magnitud y cambiando su forma a medida que aumenta la temperatura, esto se debe al decrecimiento de las constantes de tiempo de la polarización.

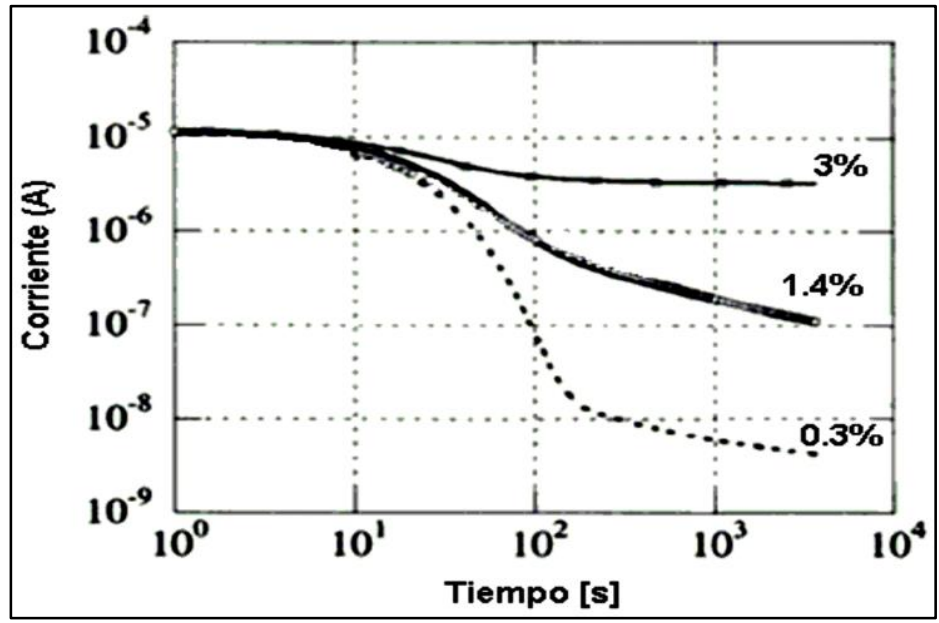

Figura 12: Variación de la corriente de polarización para varios contenidos de humedad del papel.

Fuente: Autores, (2017).

\section{II.2.2 ESTIMACIÓN DE LA CONDUCTIVIDAD}

Una de las ventajas del método PDC es que mediante la comparación de la corriente de polarización y la corriente de despolimerización se puede estimar la conductividad del aislamiento del transformador. Esto se debe a que el fenómeno de conducción sólo aparece cuando se aplica una tensión al material.

Además, mediante cálculos es posible obtener la conductividad del papel y del aceite por separado. La estimación, se basa generalmente en la comparación entre la respuesta dieléctrica medida de un transformador y la respuesta dieléctrica modelada.

Para el cálculo exacto de la conductividad, es deseable tener conocimientos completos del diseño y composición del sistema de aislamiento del transformador. Sin embargo en muchos casos, esta información no está fácilmente disponible. Para propósitos de modelado (y para la mayoría de los casos prácticos) es suficiente asumir un arreglo para el sistema de aislamiento que consta de dos electrodos cilíndricos (los arrollamientos LV y $\mathrm{HV}$ ) varias barreras cilíndricas de pressboard, ductos de aceite y separadores axiales (Figura 13).

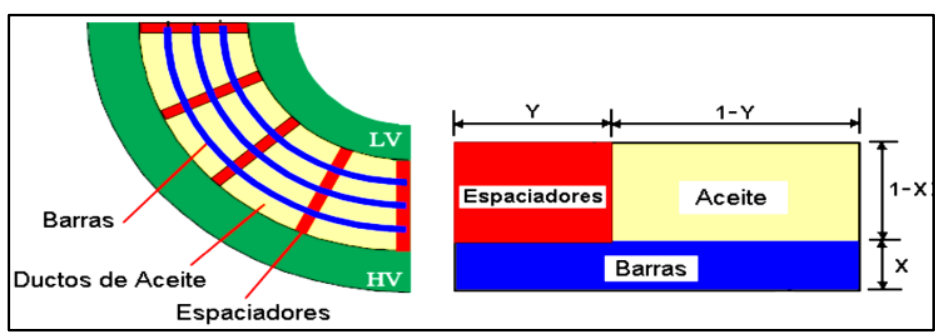

Figura 13: Modelo del sistema de aislamiento del transformador. Fuente: Autores, (2017).
Este arreglo puede ser simplificado, uniendo todas las barreras en una sola y estudiando uno de los sectores entre separadores (como se muestra en la Figura 12); adicionalmente se utiliza la cantidad relativa de separadores y barreras en el ducto, definiendo un parámetro $\mathrm{X}$ como la relación de la suma de los grosores de todas las barreras en el ducto, agrupadas y divididas por el ancho del ducto. El parámetro Y, es definido como el ancho total de todos los separadores, dividido por la longitud total de la periferia del ducto.

Generalmente las barreras llenan en un transformador del 20 al 50\% del ducto principal y los separadores llenan del 15 al $25 \%$ de la circunferencia [7].

Teniendo en cuenta este modelo, la conductividad efectiva compuesta puede ser expresada mediante la siguiente ecuación [6]:

$$
\sigma_{r}=\frac{\sigma_{\text {papel }} \cdot \sigma_{\text {acsite }}}{\sigma_{\text {papel }} \cdot(1-X)+\sigma_{\text {aceite }} \cdot X}
$$

La estimación de las conductividades del papel y el aceite, esta basada en la respuesta dieléctrica. En la referencia [6] se explica detalladamente el proceso de estimación.

\section{II.2.3 ANÁLISIS DE RESULTADOS DE PDC}

El diagnostico PDC se puede realizar:

- Ajustando un modelo sencillo X-Y, del cual se obtiene un valor numérico para la conductancia del papel y el aceite.

- Utilizando un modelo circuital equivalente del aislamiento, que permite reproducir la curva PDC.

- A través de la comparación entre dos curvas PDC obtenidas en diferentes tiempos de vida del transformador, lo que permite percibir tendencias en la degradación del aislamiento.

\section{RESPUESTA DIELÉCTRICA EN EL DOMINIO DE LA FRECUENCIA}

Cuando un voltaje senoidal, $\mathrm{u}(\mathrm{t})=\mathrm{V} \cdot \sin (\mathrm{wt})$, es aplicado en los terminales de un aislamiento, fluye una corriente total resultante que tiene dos componentes: una corriente de carga y una corriente de pérdidas. El ángulo de disipación $\delta$, esta definido como el ángulo entre el vector de la corriente total resultante y la corriente capacitiva. La tangente del ángulo $\delta$ es comúnmente conocida como el factor de disipación, la cual puede representarse como [8]:

$$
\tan \delta=\frac{I_{R}}{I C}
$$

El campo eléctrico variable aplicado, produce pequeños desplazamientos de carga que a su vez originan polarización, cuyo vector varía a la misma frecuencia del campo aplicado. Estos desplazamientos de carga originan pérdidas de potencia, las cuales evitan que el desplazamiento se encuentre en fase con el campo. Este fenómeno de polarización fuera de fase puede caracterizarse por la permitividad compleja $\varepsilon^{*}$ :

$$
\varepsilon^{*}=\varepsilon^{\prime}(w)-j \varepsilon^{\prime \prime}(w)
$$

Donde $\varepsilon^{\prime}$ y $\varepsilon^{\prime \prime}$ corresponden a la parte real e imaginaria de la permitividad compleja y son una función de la frecuencia. La parte real de la permitividad $\varepsilon^{\prime}$ representa la capacidad del 
aislamiento para almacenar energía en forma de campo eléctrico, mientras que la parte imaginaria de la permitividad $\varepsilon^{\prime \prime}$ representa las perdidas de energía del aislamiento.

Como el voltaje aplicado es una señal alterna, entonces la capacitancia medida es una cantidad compleja donde sus partes real e imaginaria, corresponden directamente a las componentes real e imaginaria de la permitividadm compleja [8]. Considerando el aislamiento como um capacitor de placas paralelas, la capacitancia compleja puede ser expresada como [8]:

$$
C=\frac{A}{d} \cdot \varepsilon^{*}=\frac{A}{d} \cdot\left(\varepsilon^{\prime}(w)-j \varepsilon^{\prime \prime}(w)\right)=C^{\prime}(w)-j C^{\prime \prime}(w)
$$

Donde, A es el área de las placas de la capacitancia, $\varepsilon^{*}$ es la permitividad compleja y "d" es la distancia entre las dos placas.

En términos de capacitancia compleja y permitividad, el factor de disipación dieléctrica (tangente $\delta$ ) puede ser expresado como [8]:

$$
\tan \delta=\frac{I_{\mathrm{R}}}{I \mathrm{C}}=\frac{C^{\prime \prime}}{C^{\prime}}=\frac{\varepsilon^{\prime \prime}(w)}{\varepsilon^{\prime}(w)}
$$

Cuando la permitividad relativa compleja es graficada en un diagrama de vectores simples, los componentes reales e imaginarios están $90^{\circ}$ fuera de fase. El vector suma $\varepsilon r^{*}$ forma un ángulo $\delta$ con el eje real $\varepsilon$ r', como se muestra en la Figura 14 [9].

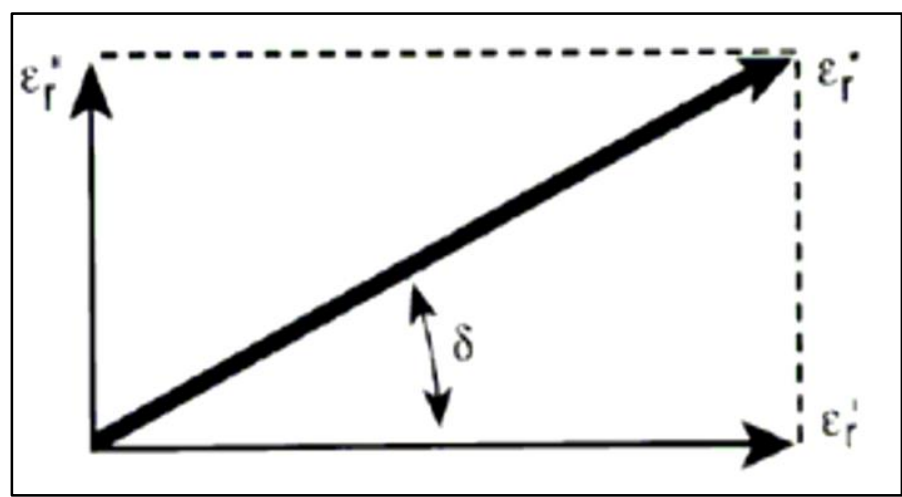

Figura 14: Diagrama Vectorial de la Permitividad compleja.

Fuente: Autores, (2017).

Además de la dependencia con la frecuencia, la permitividad compleja es dependiente de la temperatura. Un aumento de temperatura del material intensifica las pérdidas, afectando las propiedades eléctricas de todo el sistema.

\section{III.1 ESPECTROSCOPIA EN EL DOMINIO DE LA FRECUENCIA (FDS)}

En el método de espectroscopia dieléctrica en el dominio de la frecuencia (FDS, frequency domain spectroscopy) se aplica un voltaje sinusoidal (típicamente 200V RMS) de frecuencia variable, entre dos arrollamientos del transformador en un rango que va generalmente desde $0.1 \mathrm{mHz}$ a $1 \mathrm{KHz}$ y se mide la capacitancia y la tangente $\delta$.

Este método es una generalización de la medición de capacitancia y tangente $\delta$, usualmente realizada a la frecuencia de la red. La Figura 15 [8] muestra el esquema para la medición FDS, donde básicamente se obtiene la impedancia del aislamiento en un rango de frecuencia y luego se calculan la capacitancia y la tangente $\delta$.

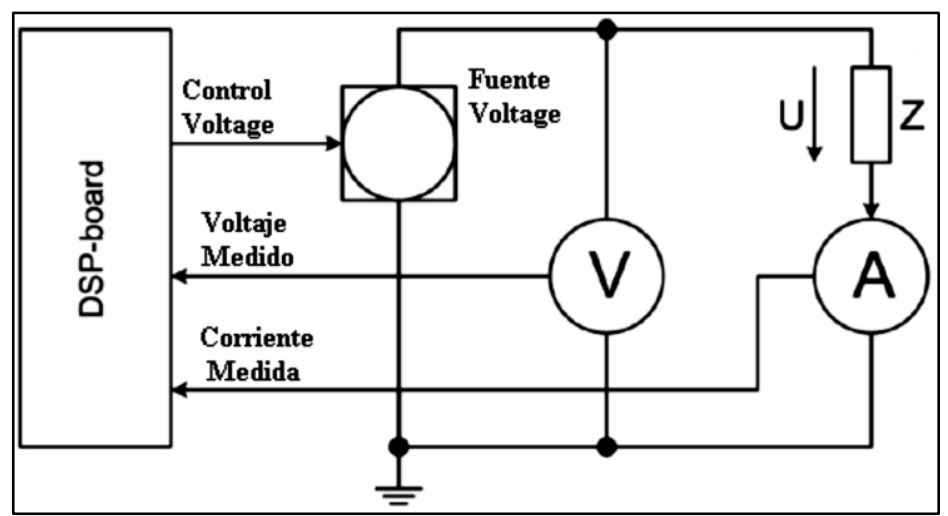

Figura 15: Circuito de medición básica FDS.

Fuente: Autores, (2017).

El voltaje y la corriente son medidos y el valor de la impedancia se calcula aplicando la ley de ohm, $\mathrm{Z}=\mathrm{V} / \mathrm{I}$.

\section{III.1.1 INFLUENCIA DE LAS PROPIEDADES DEL ACEITE Y EL PAPEL}

A través de las curvas de respuesta dieléctrica de la tangente delta, se puede acceder a la condición del aceite y del papel separadamente. En rangos de frecuencia bajos, menores a $10^{-2} \mathrm{~Hz}$ la respuesta es principalmente influenciada por las propiedades de la celulosa. Lo anterior también es cierto para rangos de frecuencia mayores a $10 \mathrm{~Hz}$.

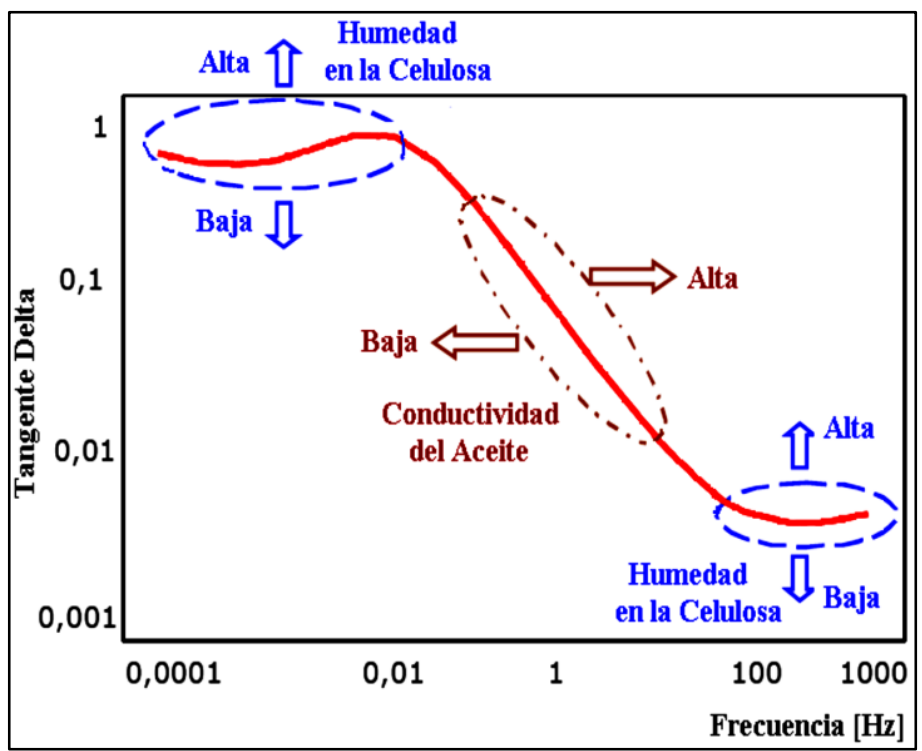

Figura 16: Influencia de las propiedades del papel y el aceite. Fuente: Autores, (2017).

Por otro lado la parte central de la respuesta está influenciada por las propiedades del aceite, principalmente por su conductividad [10][11]. En la Figura16 se muestra una curva típica de tangente delta en frecuencia.

En la Figura 17 se muestra la variación de la curva de tangente delta para 3 conductividades del aceite y para 3 contenidos de humedad del papel aislante, se pueden observar los cambios que se producen en las zonas de bajas, medias y altas frecuencias. 

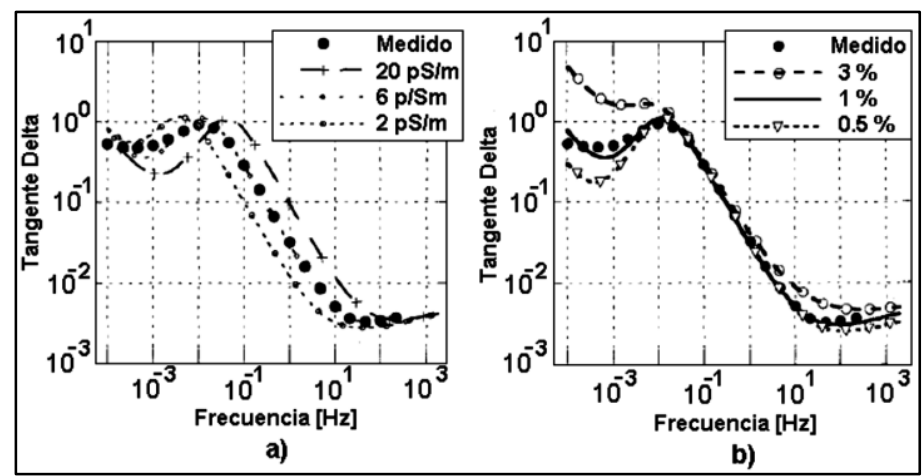

Figura 17: a) curva de $\tan \delta$ delta para 3 conductividades del aceite y b) curva para 3 contenidos de humedad del papel.

Fuente: Autores, (2017).

Como se ha indicado anteriormente, la temperatura del transformador durante la medición FDS es un parámetro muy importante, ya que su aumento causa que los factores de disipación se incrementen. El aumento de temperatura, incrementa la conductividad y aumenta la parte real de la capacitancia a bajas frecuencias (característica de dispersión de baja frecuencia).

\section{III.1.2 ESTIMACIÓN DEL CONTENIDO DE HUMEDAD}

La estimación del contenido de humedad, se basa generalmente en la comparación entre la respuesta dieléctrica medida de un transformador y la respuesta dieléctrica modelada.

Para hacer el modelado se necesita de una librería con datos sobre las propiedades dieléctricas de la celulosa a diferentes contenidos de humedad. Para propósitos de modelado (y para la mayoría de los casos prácticos) es suficiente asumir un modelo de aislamiento $\mathrm{X}-\mathrm{Y}$ que consta de varias barras cilíndricas, ductos de aceite y separadores axiales como se mostró en la Figura 12. En el dominio de la frecuencia, la permitividad dieléctrica compuesta, educt, del ducto de aislamiento es calculada como:

$$
\varepsilon(w, T)_{\text {duct }}=\frac{Y}{\frac{1-X}{\varepsilon_{\text {espac }}}+\frac{X}{\varepsilon_{\text {barras }}}}+\frac{1-Y}{\frac{1-X}{\varepsilon_{\text {aceite }}}+\frac{X}{\varepsilon_{\text {barras }}}}
$$

Donde las permitividades del aceite, espaciadores y barreras son también cantidades complejas que dependen de la frecuencia, temperatura y humedad. A partir de esta información la permitividad en función de la frecuencia del aislamiento compuesto es modelada, y comparada con los resultados de las mediciones. Actualmente existen programas comerciales para el análisis y modelamiento de la curva de respuesta dieléctrica [11].

Por otro lado, algunos autores han relacionado el contenido de humedad del papel aislante con el valor mínimo de la curva de tangente delta, a través de la ecuación empírica [8]:

$$
m . c=15.297+2.53267 \cdot \operatorname{Ln}\left(\tan \delta_{\min }\right)
$$

Donde es el contenido de humedad de la celulosa en porcentaje y $\tan \delta_{\text {min }}$ es el mínimo valor de la curva de tangente delta.

Sin embargo, a altas temperaturas $\left(70-90^{\circ} \mathrm{C}\right)$ y una frecuencia máxima de $1 \mathrm{Khz}$, el factor de disipación puede no llegar a su valor mínimo y la humedad estimada usando la ecuación empírica, podría contener cierto grado de error. Por lo que la anterior ecuación tiene una limitación a temperaturas altas en equipos de medición FDS, ya que sus frecuencias máximas están alrededor de $1 \mathrm{KHz}$. A temperaturas bajas el error que se produce al utilizar la ecuación empírica es probablemente reducido [8].

\section{III.1.3 ANÁLISIS DE RESULTADOS DE FDS}

El diagnostico FDS se puede realizar:

- Ajustando un modelo sencillo X-Y, del cual se obtiene un valor numérico para la humedad del papel y la conductancia del aceite.

- Utilizando un modelo circuital equivalente del aislamiento, que permite reproducir la curva FDS.

- A través de la comparación entre dos curvas FDS obtenidas en diferentes tiempos de vida del transformador, lo que permite percibir tendencias en la degradación del aislamiento.

\section{CONCLUSIONES}

Los métodos basados en la polarización dieléctrica RVM, PDC y FDS, pueden ayudar a evaluar la condición del aislamiento del transformador.

Por otro lado la estimación de humedad por el método RVM no es tan exacta como en los métodos PDC y FDS debido a que su esquema de interpretación es muy simple. El método RVM, puede evaluar la uniformidad del envejecimiento y de la distribución de humedad.

Los métodos PDC y FDS provén un diagnostico de la condición del aceite y del papel por separado.

\section{AGRADECIMIENTOS}

Los autores agradecen a COLCIENCIAS por su apoyo en el desarrollo de este trabajo.

\section{REFERENCIAS}

[1] M. Wang, A.J. Vandermaar y K.D. Srivastava, "Review of Condition Assessment of Power Transformers in Service", En: IEEE Electrical Insulation Magazine, Vol. 18, No. 6, (2002).

[2] A. Bognlr, G. Csqpes, L. Kalocsai y I. Kispal, "Spectrum of Polarization Phenomena of Long Time-Constant as a Diagnostic Method of oil-paper Insulating Systems", En: International Conference on Properties and Applications of Dielectric Materials ( $3^{\circ}: 1991:$ Tokyo), Proceedings of the 3rd International Conference on Properties and Applications of Dielectric Materials. Tokyo:1991.

[3] Gusztav Cscpes, Istvan Hamos, Roger Brooks y Volker Karius, "Practical Foundations of the RVM (Recovery Voltage Method for Oil/Paper Insulation Diagnosis)", En: IEEE (1998).

[4] Peter Osvath, Gusztav Csbpes y Michael Webb, "Polarisation Spectrum Analysis for Diagnosis of Insulation Systems", Tettex Instruments AG, Switzerland; Hungarian National Overvoltage Protection \& Insulation Coordination Service; Tettex Instruments Ltd, United Kingdom. 
[5] T. K. Saha, P. Purkait Member y Z. T. Yao, "Condition Monitoring of Transformer Insulation by Polarisation and Depolarisation Current Measurements", En: Australasian Universities Power Engineering Conference (2002: Melbourne, Australia).

[6] T. K. Saha y P. Purkait, "Investigation of Polarization and Depolarization Current Measurements for the Assessment of Oil-paper Insulation of Aged Transformers", En: IEEE Transactions on Dielectrics and Electrical Insulation Vol. 11, No. 1, (2004).

[7] S.M. Gubanski, P. Boss, G. Csepes, V. Der Houhanessian, J. Filippini, P. Guuinic, U. Gafvert, V. Karius, J. Lapworth, G. Urbani, P. Werelius y W. Zaeng, "Dielectric Response Methods for Diagnostics of Power Transformers", En: IEEE Electrical Insulation Magazine. Vol. 19, No. 3, (2003).

[8] J. H. Yew, T. K. Saha y A. J. Thomas, "Impact of Temperature on the Frequency Domain Dielectric Spectroscopy for the Diagnosis of Power Transformer Insulation", En: 2006 IEEE Power Engineering Society General Meeting (2006:Canada). Proceedings of the 2006 IEEE Power Engineering Society General Meeting. Montreal, Canada: 2006.

[9] C. T. Dervos, C. D. Paraskevas, P. Skafidas y P. Vassiliou, "Dielectric Characterization of Power Transformer Oils as a Diagnostic Life Prediction Method", En: IEEE Electrical Insulation Magazine. Vol. 21, No. 1, (2005).

[10] Jörgen Blennow, Chandima Ekanayake, Kzysztof Walczak, Belen Garcia, y Stanislaw M. Gubanski, "Field Experiences With Measurements of Dielectric Response in Frequency Domain for Power Transformer Diagnostics" En: IEEE transactions on Power Delivery. Vol. 21, No. 2, (2006).

[11] Maik Koch, Stefan Tenbohlen, Michael Kruger y Alexander Kraetge, "Improved Moisture Analysis of Power Transformers Using Dielectric Response Methods", En: REVUE GENERALE DE L'ELECTRICITE. France, 2008, NUMB 5, pages 62-68.

[12] Wilder Herrera, "Revision de los metodos de diagnostic en transformadores de potencia", Tesis de grado, Universidad del Valle, Escuela de Ingeniería Eléctrica y Electrónica, CaliColombia 2008.

[13] Gubanski, S.M. Boss, P. et al, "Dielectric Response Methods for Diagnostics of Power Transformers CIGRE TF15.01.09", Electra N0 202. Jun 2002. 